\title{
Wiedergelesen: Michael Balint. Der Arzt, sein Patient und die Krankheit
}

\author{
Revisited: Michael Balint. \\ The Doctor, his Patient and the Illness
}

Autor

Institut

\section{S. Ewig}

Thoraxzentrum Ruhrgebiet, Kliniken für Pneumologie und Infektiologie, Ev. Krankenhaus Herne und Augusta-Kranken-Anstalt Bochum eingereicht 30.1.2010

akzeptiert 12.2.2010

Bibliografie

Dol http://dx.doi.org/

10.1055/s-0029-1243962

Online-Publikation: 8. 3. 2010

Pneumologie 2010; 64:

278-280 @ Georg Thieme

Verlag KG Stuttgart · New York ISSN 0934-8387

\section{Korrespondenzadresse}

Prof. Dr. med. Santiago Ewig

Thoraxzentrum Ruhrgebiet Kliniken für Pneumologie und Infektiologie

Ev. Krankenhaus Herne und Augusta-Kranken-Anstalt Bochum

Bergstraße 26

44791 Bochum

ewig@augusta-bochum.de
„Februar 1983“, steht auf der Titelseite meines Exemplars von Michael Balint, „Der Arzt, sein Patient und die Krankheit“" notiert [1]. Zu dieser Zeit stand ich im letzten Drittel meines Medizinstudiums, auf der Suche nach einer Theorie der Humanmedizin. Balints Werk war das erste, das mir von diesem Thema handelte und mich begeisterte. Mein Weg führte mich in der Folge von Viktor von Weizsäcker über Franz Alexander und Alexander Mitscherlich zurück zu Sigmund Freud, von dort aus zu Thure von Uexküll. Doch alles Nach-Denken führte zu nichts; es blieb ein Schattenweg, der keine Verbindung zur Inneren Medizin erbrachte. Allenfalls die Psychiatrie schien einen Weg zum Menschen bereits gebahnt zu haben, meine Wendung zur Psychiatrie war daher vorgezeichnet. Da traf ich auf Alfred Döblins Diktum, dass er sich als „Irrenarzt“ stets wohl unter den „Irren“ gefühlt habe. Das konnte ich von mir nach meinen bisherigen Erfahrungen beim besten Willen nicht sagen. So wandte ich mich wieder der Inneren Medizin zu. Die Psychosomatik blieb eine Erinnerung an eine Gegenerzählung. Die Psychiatrie, so wurde mir rasch deutlich, hing keinesfalls einem anderen Paradigma als die Innere Medizin an. Ich aber wurde Spezialist.

Die „sieben heiligen Kühe der Psychosomatik“" wurden in der Zwischenzeit geschlachtet. Die Psychosomatik ist überwiegend zu einer Spezialdisziplin für bestimmte psychische Störungen geworden, mit denen die Psychiatrie nicht umzugehen versteht. Ihren Anspruch auf eine fachübergreifende Begründung medizinischen Handelns hat sie überwiegend aufgegeben, zumindest in

${ }^{1}$ Diese spöttische Redewendung der „sieben heiligen Kühe der Psychosomatik“ verweist auf die sieben Erkrankungen, die ihrer Theorie zufolge eine als paradigmatisch ausgegebene psychosomatische Kausalität aufwiesen, mit jeweils typischen, psychodynamisch beschreibbaren Grundkonflikten. Dazu gehörten: essenzielle Hypertonie, Asthma, Ulkuskrankheit, chronischentzündliche Darmerkrankungen, rheumatoide Arthritis, Hyperthyreose und Neurodermitis. der wissenschaftlichen Gemeinschaft nicht mehr aufrechterhalten können. Ein Blick in die Inhaltsverzeichnisse ihrer führenden Zeitschriften verrät, dass sie sich sehr weitgehend in die Erforschung psychischer Störungen bzw. physio-psychischer Interaktionen zurückgezogen hat. Balints Beitrag schließlich scheint aufgegangen in der Praxis der „Balint-Gruppen“, also der Bearbeitung der Gegenübertragungen der Therapeuten und anderer Reaktionsbildungen in Gruppen.

Liest man die ersten Seiten des Balint'schen Werkes, schlägt einem als Erstes eine Atmosphäre wie aus einer anderen Welt entgegen. Praktische Ärzte diskutieren Kasuistiken unter dem Aspekt des Gelingens der Kommunikation von Arzt und Patient und fragen nach den Gründen für ihre Störung. Die Kasuistiken enthalten längere Verläufe, die auch die Lebensumstände und die jeweilige innere Verfassung der Patienten enthalten. Allein dies, zusammen mit den Schilderungen hoffnungslos veralteter Methoden der Differenzialdiagnose und vorneuzeitlich wirkenden symptomatischen Rezepturen, versetzt den Leser in eine längst vergangene Welt. Der Kontrast zu einem zertifizierten Wirtschaftsbetrieb, wie er heute Realität ist oder zumindest immer mehr werden soll, der anhand von Standards und Behandlungspfaden Krankheiten managt, könnte größer nicht sein. Und hier bereits fällt die Entscheidung: Wollen wir von all dem nichts mehr hören, halten wir das Buch für den Ausdruck überholter und widerlegter Denkformen, oder wollen wir zumindest neugierig weiterlesen? Für mich bedarf diese Frage keines Nachdenkens.

Wir als Spezialisten geraten gleich als Erstes in die Kritik. Der Praktiker, der die Symptome seines Patienten nicht versteht und sich am Ende seiner Möglichkeiten sieht, schickt diesen zum Spezialisten. Der betreibt „Ausschlussdiagnostik“: Gleich in der ersten Kasuistik schreibt etwa ein Pneumologe in seinem Konsilbericht: „Es wird Sie freuen zu hören, dass kein Anhalt für eine Lungen- oder 
Rippenfelltuberkulose gegeben ist." Dies freut den Patienten jedoch ganz und gar nicht, denn er hat ein noch unbenanntes Problem. Praktiker und Spezialist schieben sich das ungelöste, weil unbekannte und darum unbenennbare Problem nun einander zu, es entsteht „die Verzettelung der Verantwortung“ durch die „Anonymitätsklausel“, d.h. das Versacken der Verantwortung für den Patienten zwischen Ausschlussdiagnostik und Verlegenheitsdiagnosen. Das Verhältnis von Praktiker und Spezialist ist gekennzeichnet durch die „Verewigung des Lehrer-Schüler-Verhältnisses“, d.h. die unterwürfige Haltung des Praktikers gegenüber dem scheinbar besserwissenden Spezialisten, der seinerseits aber nur eine höchst begrenzte, nämlich somatisch-spezialistische Sicht auf den Patienten hat.

Balint entwickelt hier eine klare Gegenposition. Das Verhältnis des Praktikers zum Patienten soll „eine Investierungsgesellschaft auf Gegenseitigkeit“ sein, gekennzeichnet durch ein Zuhören über lange Zeit, idealerweise über Lebensabschnitte hinweg. Das Zuhören hat eine zentrale Bedeutung in der Entschlüsselung der vorgetragenen Beschwerden, die bereits als Ergebnis einer zurückliegenden Verarbeitung von Konflikten angesehen werden, die der Patient noch vor dem ersten Aufsuchen des Arztes hinter sich gebracht hat. Sie sind vorläufige „Angebote“ an den Arzt, die er durch Annehmen zu einer anerkannten Krankheit erhebt oder aber durch Ablehnen dem Patienten zur neueren Verarbeitung zurückgibt. Mit großem Nachdruck zeichnet Balint die Bedeutung dieser Reaktion des Arztes auf das „Krankheitsangebot“ nach. Das falsche Akzeptieren des Angebots vertieft die Konfliktsituation, indem es sie „organisiert“, namentlich falsch bezeichnet und festlegt, d.h.: zur weiteren Bearbeitung unzugänglich macht; das vorzeitige Ablehnen kann seinerseits zu einer erheblichen krisenhaften Verschärfung der Konfliktsituation beitragen, da der Patient sich nun mit der Situation konfrontiert sieht, in seiner Konfliktbearbeitung von vorne anfangen zu müssen. In jedem Fall käme dem Praktiker die Aufgabe zu, zu einer „tieferen Diagnose“ vorzudringen, auf die wahren Hintergründe des Krankheitsgeschehens und -angebots einzugehen. Dabei verkennt Balint nicht, dass der Arzt hier unterscheiden lernen muss zwischen „Konflikten“ und „klinischen Krankheiten“; darüber hinaus können sich hinter „Konflikten“ auch „Grundstörungen“ verbergen. Balint ist sehr bewusst, dass nicht jeder Arzt einer solch anspruchsvollen Aufgabe gewachsen sein kann. Von Anfang an setzt er jeder Anspruchsinflation an den Arzt eine klare Grenze, auch an diejenigen, die sich der Aufgabe stellen wollen und können.

Hier kommt die „apostolische Mission“ des Arztes ins Spiel, seine Persönlichkeit, die darüber bestimmt, wie weit er in den „Konflikt“ des Patienten bzw. sogar in seine „Grundstörung“ eindringen kann bzw. sollte. Hier eröffnen sich für Balint verschiedene Techniken und Tiefengrade, die von psychotherapeutisch arbeitenden Praktikern erreicht werden können. Auch für den Spezialisten öffnen sich neue Möglichkeiten. Statt nur Ausschlussdiagnostik zu betreiben oder nichtssagende Verlegenheitsdiagnosen zu stellen, könnte er so weit kommen zu erkennen, wo seiner Meinung nach das wahre Problem liegt, und somit die Botschaft seines Konsils sehr deutlich von dem abheben, was er bisher praktiziert hat.

Balint schildert in großer Präzision die Schwierigkeiten und Fallstricke, die auf den psychotherapeutisch arbeitenden Praktiker zukommen. „Wie fängt man an?“, „Wann hört man auf“, „Was soll man behandeln?" sind die drei wichtigen Fragen in diesem Zusammenhang. Aufhören soll die Beziehung von Praktiker und Patient jedenfalls eher nicht, im Gegensatz zu einer großen Psychoanalyse, die im besten Fall nach einer langen gemeinsa- men Wegstrecke mit der Beendigung des therapeutischen Verhältnisses abgeschlossen wird. Vielmehr soll „das Gesellschaftskapital der Investierungsgesellschaft auf Gegenseitigkeit“ dauerhaft vermehrt werden. Der Praktiker jedenfalls bleibt für den Patienten eine „Droge Arzt“, wirksam mindestens wie ein Medikament oder eine operative Intervention im Guten wie im Bedenklichen. Dieser Drogenwirkung sich bewusst zu werden und diese in der Folge richtig einzusetzen, dafür glaubt Balint erste Vorarbeiten geleistet $\mathrm{zu}$ haben.

Mehr übrigens nicht. Balint wird nicht müde zu betonen, wie wenig wir wissen und wie vorläufig alle Konstruktionen sind. Weitere Forschungsarbeit wird immer wieder als unabdingbar hingestellt. Nichts an Balints Ausführungen ist dogmatisch, psychoanalytisch-spezialistisch, sektiererisch. Ganz im Gegenteil, durchweht den Text der Geist eines beweglichen Verständnisses, immer bemüht, dem Patienten besser als nur durch somatische Diagnose und „Pillen“ gerecht zu werden, wiewohl die Berechtigung auch von Placebos keineswegs durchweg in Abrede gestellt wird. „Gegenüber den vielen Fragen, die wir aufgeworfen haben, sind unsere Antworten nur sehr spärlich; vor allem haben wir dem Arzt noch kaum irgendwelche ,Ratschläge‘ zu bieten, was er zu tun und zu lassen hat, und noch weniger hat dieses Buch irgendwelche ,Beruhigung، anzubieten.“ Eindeutig scheint nur eines zu sein: „,Rat' (für den Patienten) ist gewöhnlich nur ein wohlgemeinter Schuss ins Blaue und fast immer nutzlos, und dies gilt in noch höherem Maße von jeglicher ,Beruhigung، “ Es kommt vielmehr darauf an, das Problem, den „Konflikt“ zu erkennen und zu benennen. Hier kommt ein Axiom der Psychoanalyse zum Tragen, das keinesfalls ihr Exklusivgut ist, sondern wohl ein sehr grundlegender Glaube aller Erkenntnis: Ein Problem mit Namen ansprechen zu können, heißt bereits, es prinzipiell überwunden zu haben. Das Aussprechen des Namens eines kleinen hässlichen Männleins namens „Rumpelstilzchen“ nimmt ihm bereits die Macht über die Müllerstochter.

Balints Diagnose der Medizin, im Jahre 1964, in dem dieses Buch erschien, ist eindeutig: Die Allgemeinpraxis leidet ,an einer ernstlichen, jedoch nicht bösartigen Krankheit.“ ... „Die klinische, wissenschaftliche Medizin dagegen ist keineswegs krank, sondern kerngesund und reich an Errungenschaften und Erfolgen.“

Aus dem Abstand von fast fünf Jahrzehnten erscheinen alle hier aufgeworfenen Fragen unverändert aktuell, die Vorschläge, diese zu bearbeiten, vielversprechend und haben ihre stimulierende Wirkung auf einen praktisch tätigen Arzt, sei er Praktiker oder Spezialist, nicht eingebüßt. Dennoch trennen uns Abgründe von der damaligen Situation. Den Praktiker, wie ihn sich Balint vorstellt, gibt es nicht mehr. Dies hat bekanntlich sowohl mit der Entwicklung der Medizin, der Gesundheitspolitik als auch mit der Demografie und Mobilisation der Gesellschaft zu tun. Die „Investierungsgesellschaft auf Gegenseitigkeit“, das auf Dauer gestellte Verhältnis eines Arztes zu seinem Patienten, ist weithin ein Ding der Unmöglichkeit geworden. Der Arzt kann genauso wenig wie der Pfarrer seiner „apostolischen Funktion“ in der Weise gerecht werden, wie sie hier beschrieben wird. Andererseits ist die Neigung von Medizin und Gesellschaft, auf kommunikative Probleme technische Antworten zu geben, konkret in der Medizin: technische Diagnostik einzusetzen und Medikamente zu verschreiben, statt dem Patienten zuzuhören, in der Zwischenzeit noch deutlich gewachsen. Die Probleme, die sich für Patienten daraus ergeben, sind demgegenüber nicht geringer geworden. Mein damaliger klinischer Lehrer pflegte zu sagen: „Wenn Sie heute zum Arzt gehen, sagt der Ihnen, was Sie nicht haben." Ein Blick auf unsere Arztbriefe genügt, um die Richtigkeit 
dieser Feststellung zu bestätigen. Andererseits erwarten viele Patienten eine somatische Diagnose und sind verwundert bis empört, wenn man diese nicht stellen kann. So hilft man sich als Pneumologe gerne etwa mit den Diagnosen „hyperreagibles Bronchialsystem“ oder „chronische Bronchitis“, die im besten Falle zwar tatsächlich vorliegen, aber mit den Beschwerden des Patienten, wenn überhaupt, nur bedingt etwas zu tun haben. Das Ergebnis ist, dass der Patient schließlich einen anderen Arzt aufsuchen wird. Schließlich spüren viele von uns in ihrer täglichen Praxis, dass trotz eindeutig gegebener somatischer Krankheit wichtige Dimensionen des Krankheitserlebens systematisch ausgeklammert bleiben. Balint selbst würde zugestehen, dass es Fälle gibt, wo man auch besser nicht an den „Konflikten“ bzw. „Grundstörungen“ rührt; er würde allerdings leidenschaftlich bestreiten, dass wir daran gut tun, diesen Dimensionen ausschließlich mit unserem gesunden Menschenverstand, „Rat“ oder gar „Beruhigung“ entgegenzutreten. Es wäre, wie er verschiedentlich ausführt, vergleichbar mit einem medizinischen Eingriff, der von einem Laien mit irgendeinem nicht dafür geprüften Instrumentarium ausgeführt würde.

Psychosomatisches Denken, an dieser Diagnose führt kein Weg vorbei, ist außerhalb des medizinischen Plausibilitätszusammenhangs geraten. Daran ist die Psychosomatik selbst gehörig mitschuldig. Eine der „sieben heiligen Kühe der Psychosomatik“ war das Asthma. Nach psychoanalytischer Lehre, die weitgehend zur Grundlegung der Psychosomatik herangezogen wurde, war der Konflikt des Asthmatikers typischerweise einer der Anziehung und Abstoßung, der Asthmaanfall Ausdruck eines Ambivalenzkonflikts durch erfahrene Überfürsorglichkeit und Zurückweisung. Darüber mag man heute mit einigem Recht sein Befremden ausdrücken. Eine noch gutwillige Interpretation dieser Theorie aus heutiger Sicht ergibt, dass die kleine Subgruppe der Patienten mit schwerem Asthma, überwiegend noch mit den Stigmata multipler Allergien und des Ekzemleidens versehen, als Phänotyp der Erkrankung herangezogen und manche sekundäre Krankheitsverarbeitung als Ursache des Asthmas angenommen wurde. Die Unhaltbarkeit dieser Theorie war bereits spätestens in den frühen achtziger Jahren offenkundig. Dieses Beispiel zeigt, dass die Psychosomatik schlecht beraten war, sich ausschließlich auf die Psychoanalyse zu stützen und voreilig kausale Zusammenhänge herzustellen, wo doch ihr epistemiologischer Boden nicht einmal in Ansätzen bestellt war. Der Rückzug der Psychosomatik in die Spezialität geschah allerdings zu beider Schaden, ihrer selbst und der (somatischen) Medizin.

Schauen wir einmal, was zum Thema Asthma in der aktuell gültigen Leitlinie der DGP hinsichtlich der psychosomatischen Aspekte zu lesen ist. Die Psyche findet im Zusammenhang mit Ätiologie, Differenzialdiagnose und Therapie nicht einmal Erwähnung. Lediglich bei der Aufzählung der Auslösefaktoren tauchen „psychosoziale Faktoren“ als letzte von sechs möglichen auf. Darüber hinaus wird „psychosoziale Betreuung“ als Kompenente der Asthmabehandlung aufgeführt. „Psychologische und ggf. psychotherapeutische Hilfen“ finden als Komponente der Rehabilitationsbehandlung Erwähnung. Diese Residuen sind in ihrer kraftlosen Allgemeinheit, seien wir aufrichtig, nichts anderes als Lippenbekenntnisse. Die Leitlinie gibt vielmehr sehr eindeutig der Meinung Ausdruck, dass „das Asthma“ in der Regel keiner psychotherapeutischen Behandlungsdimension bedarf.

Aus der Sicht Balints müsste man dem entgegnen, dass 1) von psychosomatischer Seite keineswegs ein prinzipieller Anspruch besteht, psychische Kausalitäten in der Pathogenese des Asthmas zu postulieren, 2) tatsächlich ein großer Teil der Asthma-Patien- ten allein durch Beachtung der in der Leitlinie zugrundegelegten Therapieprinzipien behandelbar ist, 3) demgegenüber jedoch andererseits sehr wohl viele Patienten mit gesichertem Asthma davon profitieren könnten, wenn man sich die Mühe machte, ihre Lebenssituation zu beachten und der Schilderung ihrer Probleme mit der Krankheit und durch diese hinreichend Zeit widmen würde, 4) auch bei gesichertem Asthma keineswegs immer eindeutig alle Asthma-typischen Beschwerden einer asthmatischen Aktivität entsprechen, vielmehr auch neue „Krankheitsangebote“ darstellen können, zu denen sich der Arzt wie auch immer verhalten muss, und damit zusammenhängend 5) Asthma-ähnliche Symptome, denen jedoch kein Asthma zugrundeliegt, unbedingt die erforderliche Beachtung in der Differenzialdiagnose verdienen, um „organisierte“ Stadien der Krankheitsverarbeitung zu vermeiden.

Abwegig wäre demgegenüber aus seiner Sicht der Verweis auf die grundsätzliche Priorität des Somatischen und die knappen Zeitressourcen, die einer solchen Praxis des Zuhörens entgegenstehen. Für Erstere besteht keinerlei Begründung als lediglich die Macht des Faktischen bzw. die Ökonomie; was jedoch die Zeit angeht, die als Geld angesehen wird, muss erwidert werden, dass Arzthopping, unnötige Diagnostik sowie nicht indizierte Therapie immer die für die Allgemeinheit teuerste Lösung darstellen, sodass sich sogar eine oder mehrere Stunden Gespräch immer noch als die auch ökonomisch günstigere Intervention herausstellen werden.

So gesehen erweist der Ansatz Balints unverbrauchte Inspiration und Überzeugungskraft gerade in seiner Offenheit für unterschiedliche Zugangsweisen zum Kranken, unter Auslassung voreiliger psychosomatischer Kausalitätsbehauptungen und damit Zuständigkeitsforderungen, vielmehr in seiner Sensibilität für die Mehrdimensionalität von Krankheit und Krankheitserleben. Die Zeit des Allgemeinpraktikers, wie sie Balint noch als selbstverständlich erfuhr, ist vorbei. Der Praktiker ist zum Spezialisten für das Allgemeine geworden. Somit fällt es allen Spezialisten gleichermaßen zu, die Aufgaben des Praktikers im historischen Sinne auszufüllen. Denn unverändert kennzeichnen Balints Vorstellungen von einer gelungenen Kommunikation von Arzt und Patient auch die Forderungen, die eine gute ärztliche Praxis heute ausmachen. Dazu gehören das Bewusstsein um die apostolische Funktion und ihre kritische Ausübung, die wache Analyse des „Krankheitsangebots“ des Patienten, die kompetente Unterscheidung von „Konflikt“ und (somatischer) „Krankheit“ sowie die Entschlossenheit, Verantwortung für den Patienten zu übernehmen und die „Anonymitätsklausel“ zu durchbrechen. Schließlich lohnte es durchaus der kritischen Selbstprüfung, wem von uns es gegeben ist, darüber hinaus die „Konflikte“ des Patienten näher zu benennen und diese auf dem Boden einer speziell angeeigneten Kompetenz zu bearbeiten. Viel wäre jedenfalls für die Humanmedizin gewonnen, wenn die von Balint wunderbar beschriebene Sensibilität für diese Dimension im Interesse des Patienten wieder mehr gepflegt würde.

\section{Interessenkonflikte \\ $\nabla$}

Der Autor gibt an, dass kein Interessenkonflikt besteht.

\section{Literatur}

1 Balint M. Der Arzt, sein Patient und die Krankheit. 5. Auflage. Stuttgart: Klett Cotta, 1980

2 Buhl R, Berdel D, Criée CP et al. Leitlinie zur Diagnostik und Therapie von Patienten mit Asthma. Pneumologie 2006; 60: e1 - e45 